\title{
Efficacy and Safety of Wendan Decoction for Acute Brain Injury: A Randomized Controlled Study
}

\author{
Chung-Chun Huang, MD, ${ }^{1, *}$ Pei-Yeh Chiang, MD, ${ }^{2, *}$ \\ Yu-Chen Cheng, MD, and Bor-Ren Huang, MD, PhD ${ }^{2,3}$
}

\begin{abstract}
Objectives: Despite the remarkable advances in critical care management of acute brain injury for the past 20 years, the prognoses remain poor. However, numerous reports indicate the efficacy of Traditional Chinese Medicine (TCM) therapy in stroke rehabilitation. This study aimed to determine the efficacy and safety of integrated TCM (Wendan decoction [WDD]) in patients with acute brain injury as a combination therapy in the early stages.

Design: Prospective randomized controlled trial.

Setting: Single-center study.

Subjects: Sixty patients diagnosed with acute brain injury were randomly assigned to intervention and control groups, equally, and 41 patients completed the study.

Interventions: All patients were treated by conventional neurologic intensive care. The 23 patients in the intervention group were administered with an integrated WDD in the early stages three times daily; combination treatment was initiated within 14 days and lasted $>1$ month.

Outcome measures: Duration of ventilator use, intensive care unit stays, Glasgow Coma Scale (GCS) scores, motor response, the best muscle power, disability rating scale (DRS) scores, modified Rankin scale (mRS) scores, and the mortality rate for the first month. The other outcome measures were GCS scores, motor response, the best muscle power, DRS, mRS, and Barthel index (BI) scores 6 months later.

Results: There was no mortality in the intervention group, but the rate was 39\% in the control group for first month. Comparisons between groups showed significant differences $(p<0.05)$ in GCS, DRS, mRS, and BI scores, indicating improvements in the intervention group after 6 months.

Conclusions: In the early stages of acute brain injury, combination treatment with WDD was found to be safe. Furthermore, this treatment may improve neurologic functional outcomes after 6 months.
\end{abstract}

Keywords: Chinese herbal medicine, Wendan decoction, traumatic brain injury, intracranial hemorrhage, combination therapy

\section{Introduction}

$\mathbf{T}$ HE MAJOR CAUSES of acute brain injury are traumatic brain injury (TBI) and nontraumatic intracerebral hemorrhage (ICH). The effects of cerebral injury attributed to TBI and ICH are similar. ${ }^{1}$ These two insults both carry high mortality rates and poor long-term outcomes, including vegetative status, hemiplegia, aphasia, or other neurologic deficits. Although there have been many remarkable advances in critical care management of acute brain injury for the past 20 years,${ }^{2,3}$ the prognoses of acute brain injury are still poor.

A Glasgow Coma Scale (GCS) score of 3-8 indicates a severe acute brain injury, scores from 9 to 12 signify moderate injuries, and a GCS score of 13-15 denotes mild injuries. ${ }^{4,5}$ The mortality rate is $<1 \%$ in mild brain injury cases but up to $40 \%$ in severe brain injury cases. ${ }^{5}$ The initial GCS score is the major predictor of prognosis in acute brain injury.

Departments of ${ }^{1}$ Chinese Medicine and ${ }^{2}$ Neurosurgery, Taichung Tzu Chi Hospital, Buddhist Tzu Chi Medical Foundation, Taichung, Taiwan.

${ }^{3}$ School of Medicine, Tzu Chi University, Hualien, Taiwan.

*These authors contributed equally to this work. 
For the past 30 years, the death rate from severe TBI has been reduced from $50 \%$ to $25 \%$. $^{6}$ Evidence-based guidelines for TBI management were introduced in 1995 because of varied treatment approaches, but in the years following there have still been lapses in consistent implementation. ${ }^{7,8}$ The other problem that arises when developing reliable guidelines for TBI treatment is the varied pathophysiology of injury. Most of the resultant acute and chronic complications from TBI are related to secondary injury caused by tissue damage and inflammation. ${ }^{9}$

Nontraumatic ICH is a subtype of stroke with high mortality and morbidity, with up to $50 \%$ of patients dying and half of the survivors suffering significant disabilities. ${ }^{10}$ Despite considerable research efforts in recent decades, there is still no convincing evidence of the benefits from any medical treatments; the role of surgery remains controversial and the chances of surviving ICH have not improved in developed countries over recent decades. ${ }^{11-13}$ The key factors that affect ICH outcomes are hemorrhagic volume and the hematoma itself as it can lead to secondary brain injury that causes severe neurologic deficits and death. ${ }^{14}$

Traditional Chinese Medicine (TCM) represents a large source of medical knowledge accumulated over 4000 years of observation, investigation, and clinical practice. Much knowledge has been collected in stroke treatment through this longterm medical experience. There are numerous reports about the efficacy of TCM therapies in stroke rehabilitation. ${ }^{15,16}$ However, poor study quality and bias have hindered the power of this recommendation. Wendan decoction (WDD) is one of the classical Chinese herb formulas traditionally used for gastrointestinal upset, cardiovascular disease, digestive diseases, and mental disorders. ${ }^{17,18}$ WDD was first mentioned in an ancient book of Chinese medicine (Prescriptions Worth Thousand Gold for Emergencies). In this book, it was said that WDD is used to treat some mental symptoms such as auditory hallucinations, sleep disorders, and irritability. In other studies, WDD appears to have neuroprotective effect in animal model. ${ }^{19}$ However, there are only a few studies that have integrated TCM in the treatment of patients with acute brain injury in the early stages, ${ }^{20}$ and none have included WDD. Therefore, this study is the first randomized controlled study to discuss the safety and efficacy of WDD as a combined therapy in the early stages in moderate to severe brain injury patients.

\section{Materials and Methods}

\section{Study design}

This study adopted a prospective randomized controlled design. All eligible patients included in the study agreed to participate and signed an informed consent form. In addition, the study procedures were approved by the ethical committee of Taichung Tzu Chi General Hospital (REC10326), and the authors registered the trial with ClinicalTrials.gov (NCT03851809). Patients were recruited from the neurosurgery department of Taichung Tzu Chi General Hospital between June 2014 and July 2015. A total of 60 patients were enrolled in this study and randomly assigned to either the intervention or control group. All patients in both groups were enrolled within 14 days after the episode, and a follow-up interview was performed within 6 months from the onset. The intervention group received regular neurointensive care combined with WDD, and the control group only received neurointensive care. However, 7 patients in the intervention group and 12 patients in control group did not complete the study (Fig. 1).

\section{Inclusion and exclusion criteria}

The main inclusion criteria of this study were TBI or spontaneous ICH diagnosed within 14 days of onset, an initial GCS score of 3-12 points, and adults aging between 18 and 80 years. The main exclusion criteria were a history of previous TBI or stroke, intracranial aneurysm or an arteriovenous malformation rupture, combination with another major organ injury (heart, lung, intra-abdomen organ, pelvic fracture, and major vessels), other severe diseases such as heart or kidney failure, previous cancer diagnosis, and pregnancy.

\section{Conventional neurologic intensive care}

With the guidance of the Taiwan Neurosurgical Society and the Taiwan Neurological Society, patients in the control group were given consistent treatment., $\uparrow$ The intensive treatments were established according to the TBI treatment guidelines and spontaneous ICH general treatment principles from these two societies in Taiwan.

The main guidelines for treating TBI patients included keeping vital signs stable, supplying adequate infusion volume, maintaining the intracerebral pressure (ICP) $<20 \mathrm{mmHg}$ and cerebral perfusion pressure (CPP) between 60 and $70 \mathrm{mmHg}$ (if an ICP monitor is available), ${ }^{21}$ providing early nutrition support, and performing surgical intervention based on the guideline recommendations.

In spontaneous ICH patients, the treatment principles involved keeping vital signs stable, early intubation, maintaining a systolic blood pressure (SBP) $<180 \mathrm{mmHg}$ and a diastolic blood pressure $<105 \mathrm{mmHg}$, increasing blood pressure if SBP falls $<90 \mathrm{mmHg}$, maintaining a CPP of $70 \mathrm{mmHg}$, keeping ICP $<20 \mathrm{mmHg}$, and keeping the body temperature in a normal range. Surgical intervention was needed when the size of the supratentorial hematoma became $>30$ cc combined with a GCS score $<12$ and a cerebellar hemorrhage $>3 \mathrm{~cm}$.

\section{Traditional Chinese Medicine}

In the intervention group, in addition to the conventional neurologic intensive care, WDD was given within 14 days after the episode and was used for at least 1 month. They received an average amount of $240 \mathrm{cc}$ of WDD three times daily through the feeding tube or orally depending on the patients. The WDD formula is composed of Banxia (Pinellia ternata), Shengjiang (Ginger), Bai FuLing (Poria), Zhuru (Bamboo shavings), Zhishi (ImmatureTrifoliate-orange fruit), Chenpi (Tangerine peel), and Gancao (Licorice root). The dosage of WDD ingredient herbs are as follows: Banxia 3 mace (about $11.16 \mathrm{~g}$ ), Shengjiang 1.5 mace (about $5.58 \mathrm{~g}$ ), Fuling 4 mace (about $14.88 \mathrm{~g}$ ), Zhuru 3 mace (about $11.16 \mathrm{~g}$ ), Zhishi 3 mace (about $11.16 \mathrm{~g}$ ), Chenpi 3 mace (about $11.16 \mathrm{~g}$ ), and Gancao 2 mace (about $7.44 \mathrm{~g}$ ). All the ingredient herbs were extracted with boiled water to make an aqueous extract. The composition and dosage were

\footnotetext{
*www.neurosurgery.org.tw/nsr/tbi/main.htm

${ }^{\dagger}$ www.stroke.org.tw/guideline/guideline_1.asp
} 


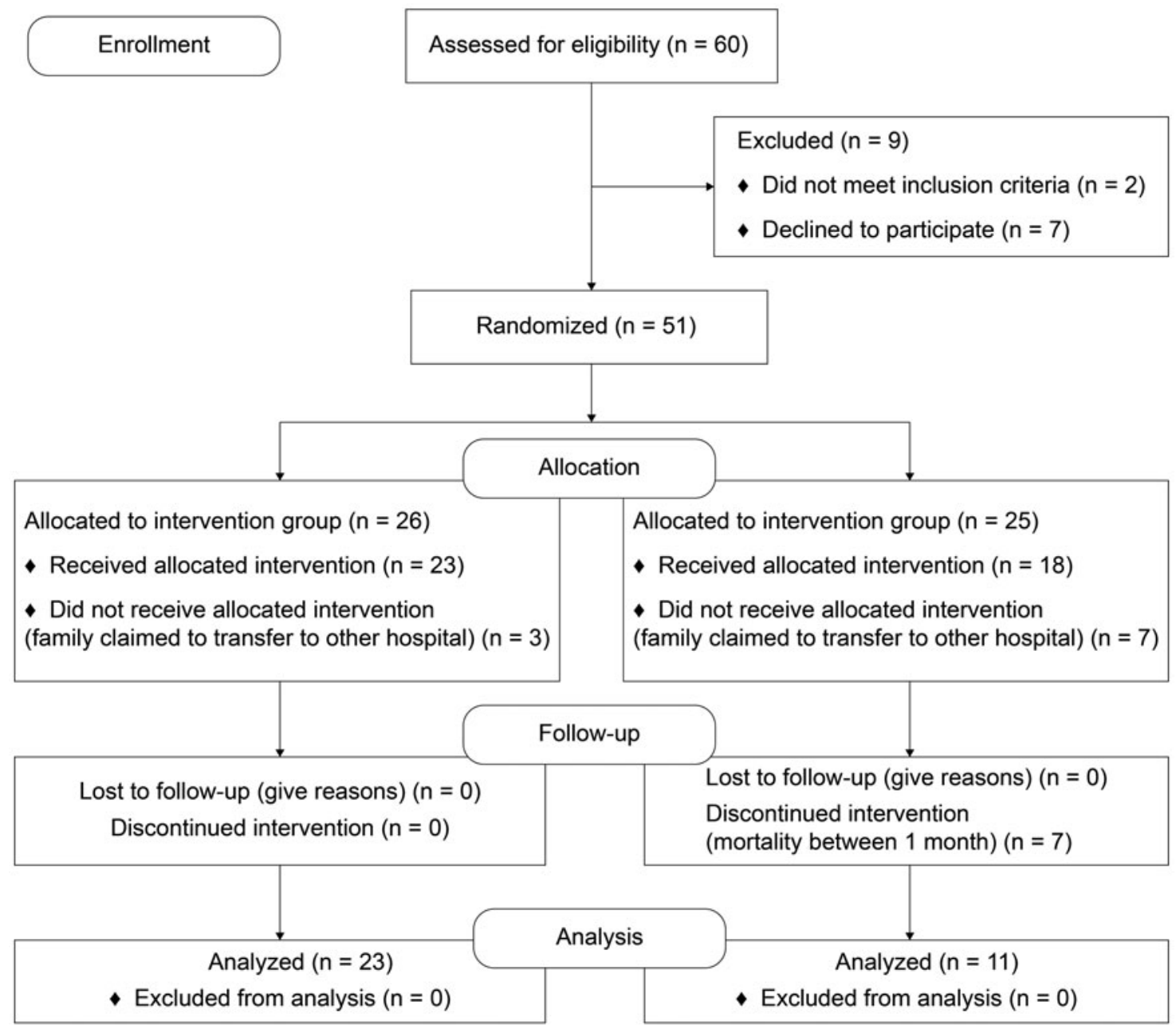

FIG. 1. Patients' flowchart.

modified according to individual condition. WDD can invigorate the spleen $(\mathrm{Pi})$, harmonize the stomach (Wei), regulate $q i$, and dispel phlegm. ${ }^{18}$

\section{Outcome measures}

The short-term (1 month after TCM use) results included GCS scores, MP values, ${ }^{4}$ the duration of ventilator use, intensive care unit (ICU) stay, disability rating scale (DRS) scores, ${ }^{22}$ modified Rankin scale (mRS) values, ${ }^{23}$ and the 1-month mortality rate. The items of the DRS are eye opening, communication ability, motor response, feeding, toilet use, grooming, level of independent function, and psychosocial adaptability; the maximum total score is 29 , and a score of 0 means nondisability and 29 means extreme vegetative status. ${ }^{24}$ The longterm (6 months after TCM use) results included GCS scores, MP scores, DRS values, mRS scores, and Barthel index (BI) values (Fig. 2). ${ }^{25}$ The BI values are commonly used to evaluate the activities of daily living. The items of the BI are feeding, bathing, grooming, dressing, bowel control, bladder control, toilet use, transfers, mobility on level surfaces, and stairs.

\section{Statistical analysis}

A Pearson's chi-square test was applied to categorical variables such as gender, craniotomy, dependent situation, and expired status. Continuous variables were analyzed using the Mann-Whitney $U$ test. A two-sided $p$-value of $<0.05$ was used as a significance threshold. All of these analyses were conducted using SPSS software (version 19, SPSS, Inc., Chicago, IL). The effect size of Cohen's $d$ score is 0.98 .

\section{Results}

\section{Baseline characteristics}

Forty-one patients completed the trial (23 patients in the intervention group and 18 in the control group; Fig. 1). The documented characteristics included age, gender, initial GCS score, motor response, best MP value, cause of brain injury, white blood cell count, and the rate of surgical intervention. No characteristics (Table 1) of the intervention group and the control group showed significant differences.

\section{Short-term results}

Table 2 summarizes the results 1 month after WDD intervention. There were seven patients in the control group who died during the month, so these characteristics were compared in the surviving participants only. There are no significant differences between the groups in ventilator use, 

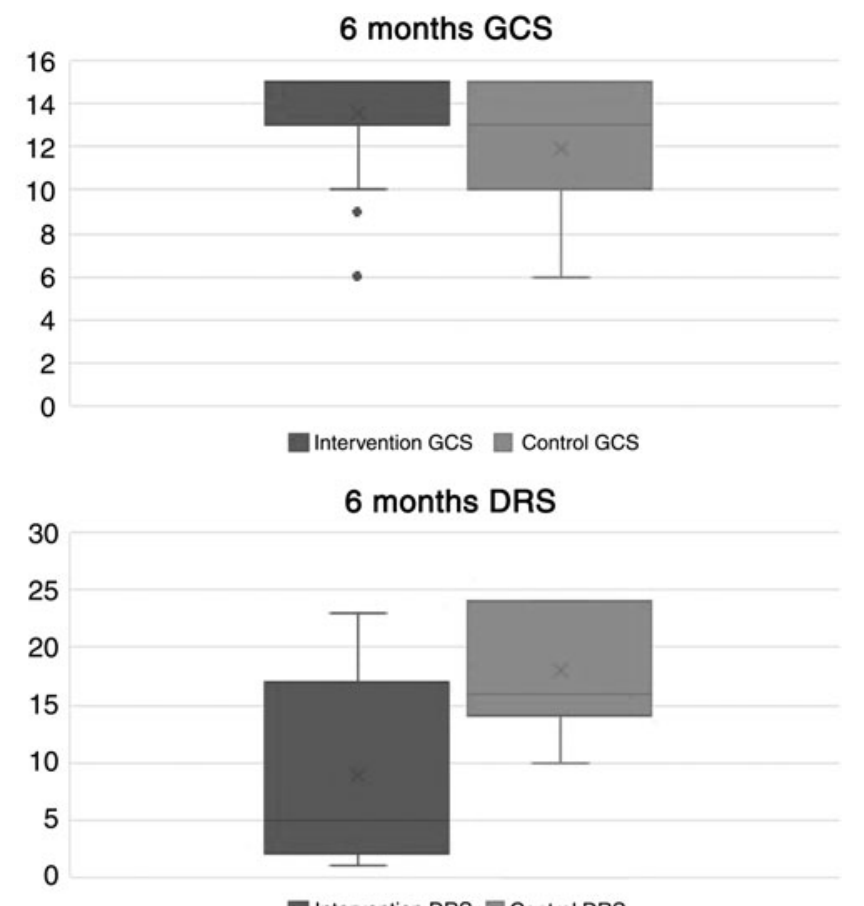

Intervention DRS $=$ Control DRS

6 months mRS
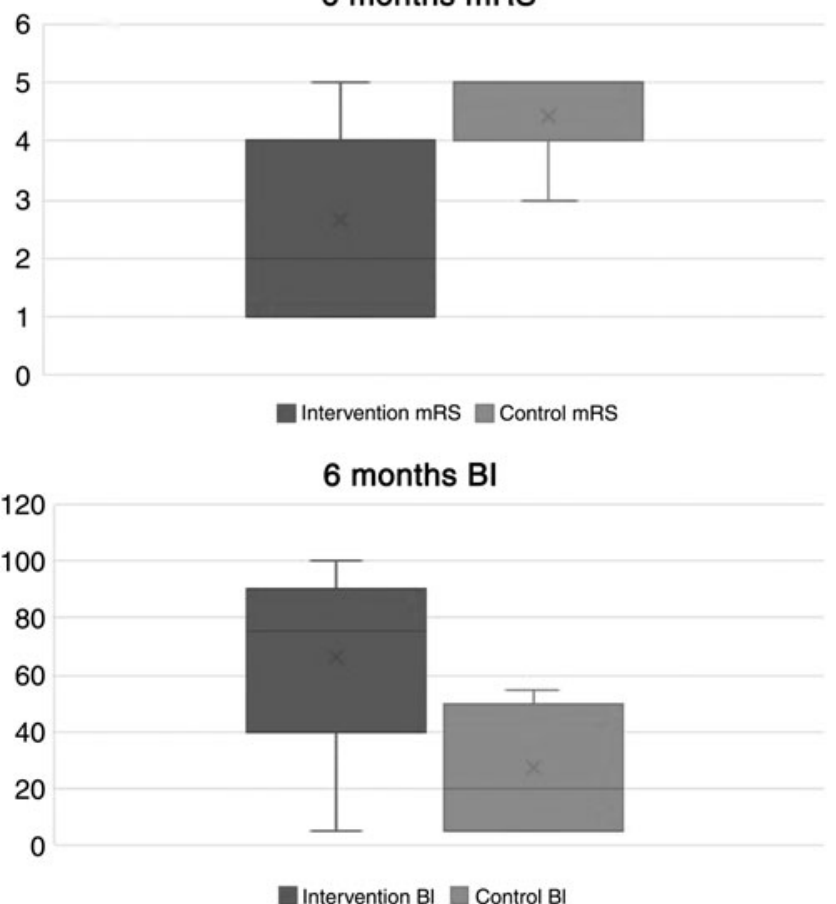

FIG. 2. The box plots of GCS, DRS, mRS, and BI after 6 months. BI, Barthel index; DRS, disability rating scale; GCS, Glasgow Coma scale; mRS, modified Rankin scale.

ICU stay, GCS values, motor responses, or MP values. However, there were differences in DRS and mRS scores between the groups; the $p$-values were 0.07 and 0.06 , respectively. Furthermore, the 1-month mortality rate was significantly different. There were no deaths during the month in the intervention group, but there was a 39\% mortality rate in the control group $(p<0.001)$.
TABle 1. Basic Characteristics

\begin{tabular}{lccc}
\hline Characteristics & $\begin{array}{c}\text { Intervention } \\
(\mathrm{n}=23)\end{array}$ & $\begin{array}{c}\text { Control } \\
(\mathrm{n}=18)\end{array}$ & $\mathrm{p}$ \\
\hline Age & $57 \pm 18$ & $59 \pm 20$ & 0.774 \\
Gender & $14(60.9)$ & $10(55.6)$ & 0.760 \\
$\quad$ Male & $9(39.1)$ & $8(44.4)$ & \\
$\quad$ Female & $7.7 \pm 2.2$ & $7.9 \pm 2.3$ & 0.743 \\
GCS & $4.5 \pm 1.4$ & $4.3 \pm 1.5$ & 0.674 \\
Motor response & $3.3 \pm 1.4$ & $2.8 \pm 1.8$ & 0.333 \\
Best muscle power & & & \\
Cause & $10(43.5)$ & $7(38.9)$ & 0.98 \\
$\quad$ Spontaneous & $13(56.5)$ & $11(61.1)$ & \\
$\quad$ Traumatic injury & $10,605 \pm 4539$ & $12,540 \pm 5436$ & 0.222 \\
WBC count & & & \\
$\quad\left(10^{3} / \mu \mathrm{L}\right)$ & $12(52.2)$ & $8(44.4)$ & 0.756 \\
Surgical intervention & $\quad 10(55.6)$ & \\
$\quad$ Yes & No & $11(47.8)$ & 10 \\
\hline
\end{tabular}

Data are presented as "mean \pm SD" or "number $(\%)$."

$* p$-Value is $<0.05$ and showed significant difference, the confidence interval is $95 \%$.

GCS, Glasgow Coma Scale; SD, standard deviation; WBC, white blood cell.

\section{Long-term results}

Table 3 shows the results 6 months after the WDD intervention. There were significant differences in GCS scores $(p=0.031), \mathrm{DRS}$ scores $(p=0.008), \mathrm{mRS}$ scores $(p=0.021)$, and BI values $(p=0.006)$ between the intervention and control groups. Conversely, there were no differences in motor response and MP values at the 6-month follow-up. The BI assessment included 10 items, and there were significant differences between feeding, toilet use, dressing, mobility, stair navigation, and transfers $(p<0.05)$.

Table 2. Results After 1 Month

\begin{tabular}{lccc}
\hline Characteristics & $\begin{array}{c}\text { Intervention } \\
(\mathrm{n}=23)\end{array}$ & $\begin{array}{c}\text { Control } \\
(\mathrm{n}=11 / 18)\end{array}$ & $\mathrm{p}$ \\
\hline $\begin{array}{l}\text { Ventilator use } \\
\quad \text { days) }\end{array}$ & $7.96 \pm 7.01$ & $10.55 \pm 9.86$ & 0.401 \\
ICU stay (days) & $12.57 \pm 7.94$ & $13.73 \pm 9.87$ & 0.72 \\
GCS & $11.8 \pm 2.9$ & $10.4 \pm 2.4$ & 0.15 \\
Motor response & $5.3 \pm 1.3$ & $5.1 \pm 0.9$ & 0.476 \\
Best muscle power & $4.0 \pm 1.1$ & $3.8 \pm 1.0$ & 0.765 \\
DRS & $16.57 \pm 7.27$ & $21.18 \pm 5.08$ & 0.07 \\
mRS & $4.2 \pm 1.0$ & $4.9 \pm 0.2$ & 0.06 \\
Mortality rate & & & $<0.001 *$ \\
$\quad$ Expired & 0 & $7(39)$ & \\
$\quad$ Survived & $23(100)$ & $11(61)$ & \\
\hline
\end{tabular}

Data are presented as "mean \pm SD" or "number (\%)"; DRS: $0=$ normal, $29=$ total independence; $\mathrm{mRS}: 0=$ no symptoms, $6=$ dead. ${ }^{*} p$-Value is $<0.05$ and showed significant difference, the confidence interval is $95 \%$.

DRS, disability rating scale; GCS, Glasgow Coma Scale; ICU, intensive care unit; mRS, modified Rankin scale; SD, standard deviation. 
Table 3. Results After 6 Months

\begin{tabular}{lccc}
\hline Characteristics & $\begin{array}{c}\text { Intervention } \\
(\mathrm{n}=23)\end{array}$ & $\begin{array}{c}\text { Control } \\
(\mathrm{n}=11)\end{array}$ & $\mathrm{p}$ \\
\hline GCS & $13.6 \pm 2.5$ & $11.9 \pm 2.2$ & $0.031^{*}$ \\
Motor response & $5.6 \pm 0.9$ & $5.4 \pm 0.7$ & 0.174 \\
Best muscle power & $4.6 \pm 0.9$ & $4.5 \pm 1.1$ & 0.670 \\
DRS & $8.8 \pm 8.7$ & $18.0 \pm 6.0$ & $0.008^{*}$ \\
mRS & $2.6 \pm 1.8$ & $4.4 \pm 0.7$ & $0.021^{*}$ \\
Barthel index & $65.9 \pm 34.9$ & $26.9 \pm 26.3$ & $0.006^{*}$ \\
Feeding & $6.7 \pm 4.4$ & $2.5 \pm 2.7$ & $0.015^{*}$ \\
Grooming & $4.1 \pm 1.9$ & $2.5 \pm 2.7$ & 0.074 \\
Toilet use & $6.5 \pm 4.4$ & $1.3 \pm 2.3$ & $0.005^{*}$ \\
Bathing & $1.3 \pm 2.2$ & 0 & 0.114 \\
Dressing & $7.2 \pm 3.3$ & $3.8 \pm 2.3$ & $0.011^{*}$ \\
Bowels & $8.5 \pm 3.2$ & $5.0 \pm 5.3$ & 0.070 \\
Bladder & $7.8 \pm 3.3$ & $4.4 \pm 4.9$ & 0.063 \\
Mobility & $8.7 \pm 6.1$ & $2.5 \pm 2.7$ & $0.015^{*}$ \\
Stair navigation & $5.0 \pm 3.7$ & 0 & $0.001^{*}$ \\
Transfers & $10.0 \pm 6.0$ & $5.0 \pm 5.3$ & $0.028^{*}$ \\
\hline
\end{tabular}

Data are presented as "mean $\pm \mathrm{SD}$ " or "number (\%)"; $p$-Values are from Mann-Whitney $U$ tests.

$* p$-Values $<0.05$ and showed significant difference, confidence interval is $95 \%$.

DRS, disability rating scale; GCS, Glasgow Coma Scale; mRS, modified Rankin scale; SD, standard deviation.

\section{Discussion}

In this study, the authors combined the WDD and conventional neurologic intensive care in the early stages of acute brain injury and tested its safety and efficacy. In addition, they also designed the study as a prospective and randomized controlled trial. To the best of their knowledge, this study is the first randomized controlled clinical trial of WDD in acute brain injury. The authors found that WDD integrated with conventional neurologic intervention can significantly improve neurologic function within half a year of an acute brain injury.

There were no severe complications in the intervention group, but a few patients experienced mild diarrhea. All of them improved after adjusting to the dose of WDD. Moreover, there was no mortality in the intervention group. Although there was a significant difference between the two groups in the 1-month mortality rate, the authors consider these results only demonstrate the safety of WDD intervention in the early stage. Seven patients of control group died within 1 month. Between them, age ranged from 18 to 80 and mean is 62, initial GCS ranged from 3 to 15 and mean is $8.2,4$ is male and 3 is female, 2 is spontaneously ICH and 5 is TBI, and 3 patients were performed surgery. These characteristics are not significantly different.

In the results 1 month after brain injury of WDD intervention, DRS $(p=0.07)$ and $\operatorname{mRS}(p=0.06)$ values showed differences, but they were not significant. These results indicate that TCM intervention in the early stages may improve the neurologic functional outcome because the $p$-value is near 0.05 , even with the low number of patients.

There is no difference in the duration of ventilator use and ICU stay between groups, $(p=0.401$ and $p=0.72)$. Notably, there are many other factors (such as heart and lung function) that influence the ventilator use and ICU stay. Likewise, there were no differences in GCS scores, motor response, and best MP values.

Six months after WDD intervention, GCS, DRS, mRS, and BI values all showed significant differences. It is well known that $\mathrm{mRS}$ and $\mathrm{BI}$ values represent long-term neurologic functional outcomes. ${ }^{26,27}$ Overall, these results suggest that WDD integrated with conventional neurologic intensive care in the early stages of acute brain injury could improve neurologic functional outcomes, especially after 6 months. Furthermore, WDD improved feeding, toilet use, dressing, mobility, stair navigation, and transfers in patients.

Acute brain injury includes TBI and nontraumatic ICH with high mortality and poor neurologic outcomes. In addition to surgical interventions, there have been many advancements in neurointensive care in the past 20 years; however, the outcomes are still unsatisfactory because secondary injury cannot be treated completely. ${ }^{5}$

The effects of cerebral injury attributed to TBI and ICH are similar. ${ }^{1}$ The complex pathogenesis of acute brain injury is initially induced by mechanical damage, followed by a series of secondary injury cascades. ${ }^{28}$ During the acute and chronic stages of brain injury, neuroinflammation has been implicated as the key to pathology and treatment. ${ }^{29}$ There are many experimental studies suggesting TCM provides neuroprotection in brain injury through an anti-inflammatory pathway. ${ }^{30}$

WDD has been shown to protect against neuronal damage in rats by inhibiting inflammatory cytokines ${ }^{19,31}$ and offer clinical benefits in the treatment of schizophrenia. ${ }^{17,18}$ Biochemically, WDD may increase the ability of learning and memory through improving the content and function of central glutamate, and preventing or delaying the degeneration of hippocampal neuronal cells. ${ }^{32}$ It is well known that secondary injury and neuroinflammation are major unsolved problems in acute brain injury; inflammatory cytokines are considered as phlegm stasis in TCM. After a brain injury, overall $q i$ circulation is impaired; the resulting decrease in the ability to remove phlegm stasis in the brain is harmful to neuron repair. Furthermore, this situation becomes a vicious cycle. Therefore, the authors hypothesized that removing phlegm stasis, and thus restoring the human body's energy operation system, could accelerate the repair processes in the brain. Prescriptions at acute stages were based on modified WDD to decrease the inflammatory cytokines by restoring human $q i$ circulation. Once $q i$ circulation is recovering, the authors can typically see patients' clinical symptoms and digestion status improve 7-10 days after initiating WDD.

A limitation of this study is the low patient's number. Specifically, there were only 11 cases remaining in the control group after 6 months. Hence, statistical bias cannot be excluded. The authors have planned another study that will include more patients with acute brain injury to confirm these results.

\section{Conclusion}

This study combined WDD and conventional neurointensive care in acute brain injury, resulting in improved outcomes and using the WDD in the early stages of acute brain injury may be safe and effective.

\section{Author Disclosure Statement}

No competing financial interests exist. 


\section{Funding Information}

This study was supported by Taichung Tzu Chi General Hospital (grant no. TTCRD103-27). The funding agency had no role in the study design, the collection, analysis, or interpretation of data, the writing of the report, or the decision to submit the article for publication.

\section{References}

1. Powers WJ. Intracerebral hemorrhage and head trauma: Common effects and common mechanisms of injury. Stroke 2010;41(10 Suppl):S107-S110.

2. Bullock R, Chesnut RM, Clifton G, et al. Guidelines for the management of severe head injury. Brain Trauma Foundation. Eur J Emerg Med 1996;3:109-127.

3. Bullock MR, Povlishock JT. Guidelines for the management of severe traumatic brain injury. Editor's Commentary. J Neurotrauma 2007;24 Suppl 1:2 p preceding S1.

4. Teasdale G, Jennett B. Assessment of coma and impaired consciousness. A practical scale. Lancet 1974;2:81-84.

5. Maas AI, Stocchetti N, Bullock R. Moderate and severe traumatic brain injury in adults. Lancet Neurol 2008;7:728-741.

6. Lu J, Marmarou A, Choi S, et al. Mortality from traumatic brain injury. Acta Neurochir Suppl 2005;95:281-285.

7. Ghajar J, Hariri RJ, Narayan RK, et al. Survey of critical care management of comatose, head-injured patients in the United States. Crit Care Med 1995;23:560-567.

8. Hesdorffer DC, Ghajar J, Iacono L. Predictors of compliance with the evidence-based guidelines for traumatic brain injury care: A survey of United States trauma centers. J Trauma 2002;52:1202-1209.

9. Hanna JS, Gracias VH. Nonoperative management of splenic trauma should be approached with caution in the setting of traumatic brain injury. J Trauma Acute Care Surg 2014;76:1334.

10. van Asch CJ, Luitse MJ, Rinkel GJ, et al. Incidence, case fatality, and functional outcome of intracerebral haemorrhage over time, according to age, sex, and ethnic origin: A systematic review and meta-analysis. Lancet Neurol 2010;9:167-176.

11. Adeoye O, Woo D, Haverbusch M, et al. Surgical management and case-fatality rates of intracerebral hemorrhage in 1988 and 2005. Neurosurgery 2008;63:1113-1118.

12. Zahuranec DB, Lisabeth LD, Sánchez BN, et al. Intracerebral hemorrhage mortality is not changing despite declining incidence. Neurology 2014;82:2180-2186.

13. Wei JW, Huang Y, Wang JG, et al. Current management of intracerebral haemorrhage in China: A national, multicentre, hospital register study. BMC Neurol 2011;11:16.

14. Qureshi AI, Tuhrim S, Broderick JP, et al. Spontaneous intracerebral hemorrhage. N Engl J Med 2001;344:1450-1460.

15. Zhang Y, Jin H, Ma D, et al. Efficacy of integrated rehabilitation techniques of traditional Chinese medicine for ischemic stroke: A randomized controlled trial. Am J Chin Med 2013;41:971-981.

16. Xu S, Pang Q, Lin Z, Zhang N. Effect of integrated traditional Chinese and Western medicine therapy for acute hypertensive intracerebral hemorrhage: A meta-analysis. Artif Cells Nanomed Biotechnol 2017;45:1-6.

17. Deng $\mathrm{H}, \mathrm{Xu}$ J. Wendan decoction (Traditional Chinese medicine) for schizophrenia. Cochrane Database Syst Rev 2017;6:CD012217.

18. Wieland LS, Santesso N. Summary of a Cochrane review: Wendan decoction traditional Chinese medicine for schizophrenia. Eur J Integr Med 2017;15:81-82.
19. Li YB, Zhang WH, Liu HD, et al. Protective effects of Huanglian Wendan decoction aganist cognitive deficits and neuronal damages in rats with diabetic encephalopathy by inhibiting the release of inflammatory cytokines and repairing insulin signaling pathway in hippocampus. Chin $\mathbf{J}$ Nat Med 2016;14:813-822.

20. Gau BS, Yang HL, Huang SJ, Lou MF. The use of complementary and alternative medicine for patients with traumatic brain injury in Taiwan. BMC Complement Altern Med 2012;12:211.

21. Koskinen LO, Olivecrona M, Grände PO. Severe traumatic brain injury management and clinical outcome using the Lund concept. Neuroscience 2014;283:245-255.

22. Rappaport M, Hall KM, Hopkins K, et al. Disability rating scale for severe head trauma: Coma to community. Arch Phys Med Rehabil 1982;63:118-123.

23. Wilson JT, Hareendran A, Grant M, et al. Improving the assessment of outcomes in stroke: Use of a structured interview to assign grades on the modified Rankin Scale. Stroke 2002;33:2243-2246.

24. Neese LE, Caroselli JS, Klaas P, et al. Neuropsychological assessment and the Disability Rating Scale (DRS): A concurrent validity study. Brain Inj 2000;14:719-724.

25. Mahoney FI, Barthel DW. Functional evaluation: The Barthel index. Md State Med J 1965;14:61-65.

26. Howard G, Waller JL, Voeks JH, et al. A simple, assumption-free, and clinically interpretable approach for analysis of modified Rankin outcomes. Stroke 2012;43: 664-669.

27. Duffy L, Gajree S, Langhorne P, et al. Reliability (interrater agreement) of the Barthel Index for assessment of stroke survivors: Systematic review and meta-analysis. Stroke 2013;44:462-468.

28. Sheriff FG, Hinson HE. Pathophysiology and clinical management of moderate and severe traumatic brain injury in the ICU. Semin Neurol 2015;35:42-49.

29. Corps KN, Roth TL, McGavern DB. Inflammation and neuroprotection in traumatic brain injury. JAMA Neurol 2015;72:355-362.

30. Xing Z, Xia Z, Peng W, et al. Xuefu Zhuyu decoction, a traditional Chinese medicine, provides neuroprotection in a rat model of traumatic brain injury via an anti-inflammatory pathway. Sci Rep 2016;6:20040.

31. Jia KK, Ding H, Yu HW, et al. Huanglian-Wendan decoction inhibits NF-kappaB/NLRP3 inflammasome activation in liver and brain of rats exposed to chronic unpredictable mild stress. Mediators Inflamm 2018;2018:3093516.

32. Zhu JH, Sun Hx, Xiong QY, et al. Effect of Wendan Decoction on contents of serum TNF- $\alpha$, IL- 6 and expression of Glu in hippocampus tissue in rats with schizophrenia. Clin J Exp Tradit Med Formul 2014;20:160-164.

Address correspondence to: Bor-Ren Huang, MD, PhD Department of Neurosurgery Taichung Tzu Chi Hospital

Buddhist Tzu Chi Medical Foundation No 88, Sec 1, Fengxing Road Tanzi District Taichung City 427 Taiwan

E-mail: smallblue525@hotmail.com 